\title{
On two categories of many valued relations
}

\author{
Dikran Dikranjan ${ }^{1}$ Alexander Šostak ${ }^{2}$ \\ ${ }^{1}$ Department of Mathematics and Informatics, University of Udine, Italy \\ ${ }^{2}$ Institute of Mathematics and Computer Science, University of Latvia, Riga, Latvia
}

\begin{abstract}
The purpose of these notes is to introduce the concept of a many-valued relation and to study two, in a certain sense dual, categories of such relations. Besides, we apply the concept of a manyvalued relation to the description of different approaches, both fixed-based and variable-based, to Fuzzy Topology and characterize categories related to Fuzzy Topology, in the framework of the corresponding categories of many-valued relations.
\end{abstract}

Keywords: $L$-fuzzy relation, many-valued relation, fuzzy topology, closure operator, interior operator

\section{Introduction}

Basing on the concept of an $L$-fuzzy relation between elements of two sets (see e.g. [19], [17], [4]), we introduce the notion of a many-valued relation and define two, in a certain sense dual, categories, $\mathcal{M R E L}$ and $\mathcal{N}$ REL, whose objects are such relations. Our special interest is in $L$-valued relations between elements of a set and $L$-fuzzy subsets of this set. We distinguish two classes of such relations, calling them expansive and inclusive respectively. Expansive and inclusive many-valued relations are used to characterize different categories related to fuzzy topology, both fixed-based [8] and variable-based [12]. The first ones, expansive, allow us to develop the closure operator based approach to the subject of Fuzzy Topology, while inclusive $L$-valued relations lead to an alternative, that is interior operator based approach. We suppose that the presented here approach to Fuzzy Topology in the framework of many-valued relations will allow to get an additional insight into its subject.

\section{Two categories of many-valued relations}

In this Section we define two categories. The objects of the both categories are many-valued relations. The difference of the categories is in the way how we define their morphisms. The first one of these categories, MREL, as a prototype, has the category of topological spaces (and more general, variablebased fuzzy topological spaces) defined by closure operators, while the second one, $\mathcal{N} \mathbf{R E L}$ as a prototype, also has the category of topological spaces (and more general, variable-based fuzzy topological spaces), however in this case defined by interior operators.

\section{1. $L$-relations}

Let $L=(L, \leq \vee, \wedge)$ be a frame (see e.g. [6]), that is a complete infinitely distributive lattice (that is $\beta \wedge\left(\bigvee_{i} \alpha_{i}\right)=\bigvee_{i}\left(\beta \wedge \alpha_{i}\right)$ holds for any $\left\{\alpha_{i}: i \in\right.$ I\} $\subset L, \beta \in L)$. The bottom and the top elements of the frame $L$ are denoted by $0_{L}$ and $1_{L}$ respectively. Let FRM be the category of frames. The objects of this category are frames and morphisms from a frame $L=\left(L, \leq_{L} \vee_{L}, \wedge_{L}\right)$ to a frame $L^{\prime}=\left(L^{\prime}, \leq_{L^{\prime}} \vee_{L^{\prime}}, \wedge_{L^{\prime}}\right)$ are mappings $\varphi: L \rightarrow L^{\prime}$ which preserve finite meets and arbitrary joins, see e.g. [6]. In particular, this means that $\varphi\left(0_{L}\right)=0_{L^{\prime}}$ and $\varphi\left(1_{L}\right)=1_{L^{\prime}}$.

The following, fundamental for us, concept is well-known and appears (under different names) in many papers see e.g. [19], [4], [1], [2], [17].

Definition 2.1 An L-relation, or an L-fuzzy relation, on the product $X \times Y$ is a mapping $R$ : $X \times Y \rightarrow L$. In case the frame $L$ is not specified, we refer to an L-relation just as a "many-valued" relation.

Let an $L$-fuzzy relation $R: X \times Y \rightarrow L$ be given. Then for each $x \in X$ this $L$-fuzzy relation generates an $L$-fuzzy set $R_{x}: Y \rightarrow L$ defined by $R_{x}(y)=$ $R(x, y)$. Similarly, for each $y \in Y$ it generates an $L$ fuzzy set $R_{y}: X \rightarrow L$ defined by $R_{y}(x)=R(x, y)$.

Given an $L$-relation $R: X \times Y \rightarrow L$ its inverse $L$ relation $R^{-1}: Y \times X \rightarrow L$ is defined by $R^{-1}(y, x)=$ $R(x, y)$ for all $x \in X, y \in Y$.

\subsection{The category MREL of many-valued relations.}

Definition 2.2 (cf. [14], [15]) The objects of the category MREL are many-valued relations, that is quadruples $(X, Y, L, R)$ where $X, Y$ are sets, $L$ is a frame and $R$ is an L-fuzzy relation on $X \times Y$. The morphisms $F:(X, Y, L, R) \rightarrow\left(X^{\prime}, Y^{\prime} L^{\prime}, R^{\prime}\right)$ are triples $(u, v, \varphi)$ where $u: X \rightarrow X^{\prime}, v: Y \rightarrow Y^{\prime}$ are mappings and $\varphi: L \rightarrow L^{\prime}$ is a morphism in the category FRM, such that $\varphi(R(x, y)) \leq R^{\prime}(u(x), v(y))$ for all $x \in X$ and $y \in Y$ :

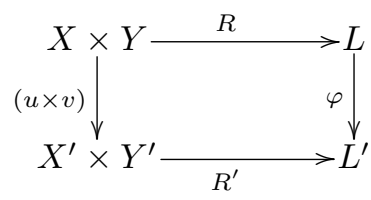


Example 2.3 Let $L=L^{\prime}=2=\{0,1\}$ be the two-point lattices and let $\varphi=i d_{2}$ be the identity mapping on 2. Notice that

$$
R(x, y)=1 \Longleftrightarrow(x, y) \in R
$$

and

$$
R^{\prime}(u(x), v(y))=1 \Longleftrightarrow(u(x), v(y)) \in R^{\prime} .
$$

Hence $F=(u, v, \varphi):(X, Y, R, 2) \rightarrow\left(X^{\prime}, Y^{\prime}, R^{\prime}, 2\right)$ is a morphism in the category MREL if and only if $(u \times v)(R) \subseteq R^{\prime}$.

Lemma 2.4 The following properties are equivalent:

(a) The triple $F=(u, v, \varphi): R \rightarrow R^{\prime}$ is a morphism in MREL;

(b) $\varphi \circ R_{x} \leq R_{u(x)}^{\prime} \circ v$ holds for every $x \in X$;

(c) $\varphi \circ\left(v\left(R_{x}\right)\right) \leq R_{u(x)}^{\prime}$ for each $x \in X$.

Proof $(a) \Longleftrightarrow(b)$ The equivalence of (a) and (b) can be established as follows:

$$
\begin{gathered}
\varphi \circ R_{x} \leq R_{u(x)}^{\prime} \circ v \Longleftrightarrow \\
\Longleftrightarrow\left(\varphi \circ R_{x}\right)(y) \leq\left(R_{u(x)}^{\prime}(v(y)) \forall y \in Y \Longleftrightarrow\right. \\
\Longleftrightarrow \varphi\left(R_{x}(y)\right) \leq R^{\prime}(u(x), v(y)) \forall y \in Y \Longleftrightarrow \\
\Longleftrightarrow \varphi(R(x, y)) \leq R^{\prime}(u(x), v(y)) \quad \forall y \in Y .
\end{gathered}
$$

Thus the condition $\varphi \circ R_{x} \leq R_{u(x)}^{\prime} \circ v$ holds for all $x \in X$ if and only if $F=(u, v, \varphi): R \rightarrow R^{\prime}$ is a morphism in the category MREL.

$(a) \Longrightarrow(c)$ Assume now that $F=(u, v, \varphi): R \rightarrow$ $R^{\prime}$ is a morphism in MREL and fix $x \in X$. Then for every $y^{\prime} \in Y$ :

$$
\begin{gathered}
\varphi\left(v\left(R_{x}\right)\left(y^{\prime}\right)\right)=\varphi\left(\sup _{v(y)=y^{\prime}} R_{x}(y)\right)= \\
\sup _{v(y)=y^{\prime}} \varphi(R(x, y)) \leq \\
\sup _{v(y)=y^{\prime}} R^{\prime}(u(x), v(y))=R^{\prime}\left(u(x), y^{\prime}\right)=R_{u(x)}^{\prime}\left(y^{\prime}\right),
\end{gathered}
$$

that is $\varphi \circ\left(v\left(R_{x}\right)\right) \leq R_{u(x)}^{\prime}$ for each $x \in X$.

$(c) \Longrightarrow(b)$ Assume that $\varphi \circ\left(v\left(R_{x}\right)\right) \leq R_{u(x)}^{\prime}$ for each $x \in X$. We have to show that $\left(\varphi \circ R_{x}\right)(y) \leq$ $\left(R_{u(x)}^{\prime} \circ v\right)(y)$ for each $y \in Y$.

Fix any $y_{0} \in Y$ and let $y_{0}^{\prime}=v\left(y_{0}\right)$. Then according to our assumption

$$
\varphi\left(v\left(R_{x}\right)\left(y_{0}^{\prime}\right)\right) \leq R_{u(x)}^{\prime}\left(y_{0}^{\prime}\right) .
$$

Noticing that

$$
v\left(R_{x}\right)\left(y_{0}^{\prime}\right)=\sup _{v(y)=y_{0}^{\prime}} R_{x}(y) \geq R_{x}\left(y_{0}\right),
$$

we conclude, from the above inequality, that

$$
\left(\varphi \circ R_{x}\right)\left(y_{0}\right) \leq R_{u(x)}^{\prime}\left(y_{0}^{\prime}\right)=\left(R_{u(x)}^{\prime} \circ v\right)\left(y_{0}\right)
$$

for all $y_{0} \in Y$, and $x \in X$

\subsection{The category $\mathcal{N R E L}$ of many-valued relations}

Definition 2.5 The objects of the category $\mathcal{N R E L}$ are many-valued relations, that is quadruples $(X, Y, L, R)$ where $X, Y$ are sets, $L$ is a frame and $R$ is an $L$-fuzzy relation on $X \times Y$. The morphisms $F:(X, Y, L, R) \rightarrow\left(X^{\prime}, Y^{\prime} L^{\prime}, R^{\prime}\right)$ are triples $(u, v, \psi)$ where $u: X \rightarrow X^{\prime}, v: Y^{\prime} \rightarrow Y$ are mappings and $\psi: L^{\prime} \rightarrow L$ is a morphism in the category FRM, such that $\psi R^{\prime}\left(u(x), y^{\prime}\right) \leq R\left(x, v\left(y^{\prime}\right)\right)$ for all $x \in X$ and $y^{\prime} \in Y$ :

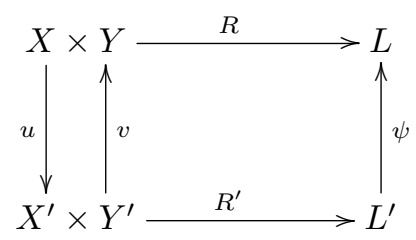

Example 2.6 Let $L=L^{\prime}=2$ be the two-point lattice and $\psi=i d_{2}$ be the identity mapping. Notice that the inequality $\psi R^{\prime}\left(u(x), y^{\prime}\right) \leq R\left(x, v\left(y^{\prime}\right)\right)$ in this case just means:

$$
\left(u(x), y^{\prime}\right) \in R^{\prime} \Longrightarrow\left(x, v\left(y^{\prime}\right)\right) \in R,
$$

and hence this property characterizes those $F=(u, v, 2, i d):(R \subseteq X \times Y) \rightarrow\left(R^{\prime} \subseteq X^{\prime} \times Y^{\prime}\right)$ which are morphisms in the category $\mathcal{N}$ REL.

Lemma 2.7 The triple $F=(u, v, \psi): R \rightarrow R^{\prime}$ is a morphism in $\mathcal{N R E L}$ if and only if $\psi \circ R_{y^{\prime}}^{\prime} \circ u \leq$ $R_{v\left(y^{\prime}\right)}$ for each $y^{\prime} \in Y^{\prime}$.

Proof. It suffices to note that for all $x \in X$ one has

$$
\begin{gathered}
\psi \circ R_{y^{\prime}}^{\prime} \circ u \leq R_{v\left(y^{\prime}\right)} \Longleftrightarrow \\
\left(\left(\psi \circ R_{y^{\prime}}^{\prime} \circ u\right)(x) \leq R\left(x, v\left(y^{\prime}\right)\right) \forall x \in X \Longleftrightarrow\right. \\
\left(\psi \circ R_{y^{\prime}}^{\prime}\right)(u(x)) \leq R\left(x, v\left(y^{\prime}\right)\right) \forall x \in X \Longleftrightarrow \\
\psi\left(R^{\prime}\left(u(x), y^{\prime}\right) \leq R\left(x, v\left(y^{\prime}\right)\right) .\right.
\end{gathered}
$$

\section{The category of many-valued closure spaces as a category of many-valued relations}

\section{1. $L$-fuzzy closure spaces}

As before, let $L=(L, \leq, \wedge, \vee)$ be a frame. The following concepts are well known and appear in many works, however, often under different names, see e.g. [6], [3], [7], [5], [9], [13], etc.

Definition 3.1 An (L-fuzzy) closure operator on the $L$-powerset $L^{X}$ of a set $X$ is a mapping $c$ : $L^{X} \rightarrow L^{X}$ such that

(1) $c\left(0_{X}\right)=0_{X}$ where $0_{X}: X \rightarrow L$ is the constant mapping with value $0_{L}$;

(2) $c(A) \geq A$ for every $A \in L^{X}$;

(3) $c(A) \leq c(B)$ whenever $A \leq B, A, B \in L^{X}$. 
An (L-fuzzy) closure operator c : $L^{X} \rightarrow L^{X}$ is called pretopological if it satisfies condition

(4) $c(A \vee B)=c(A) \vee c(B)$

An (L-fuzzy) pretopological closure operator $c$ : $L^{X} \rightarrow L^{X}$ is called topological if it satisfies condition

(5) $c(c(A))=c(A)$ for every $A \in L^{X}$.

The pair $(X, c)$ is called respectively an L-fuzzy closure space, an L-fuzzy closure pretopological space, and an L-fuzzy closure topological space. In case when the frame $L$ is not specified we speak about many-valued closure operators, many-valued closure spaces, etc.

To define the category whose objects are arbitrary many-valued closure spaces and its subcategories of many-valued pretopological closure spaces and many-valued closure topological spaces we have to specify morphisms between such spaces. In order to define morphisms in these categories we need, as before, two frames. However, now we prefer to denote them by $L$ and $M=\left(M, \leq_{M}, \wedge_{M}, \vee_{M}\right)$ respectively.

Given a mapping $F: L^{X} \rightarrow L^{Y}$, where $X, Y$ are sets, and a function $\varphi: L \rightarrow M$, we call image of $F$ under $\varphi$ the function $\varphi(F): L^{X} \rightarrow M^{Y}$ defined by $\varphi(F)(A)(y)=\varphi(F(A)(y))$ for every $A \in L^{X}$ and $y \in Y$. Further, recall that given a mapping $f$ : $X \rightarrow Y$, its forward $L$-powerset lift $f \rightarrow: L^{X} \rightarrow L^{Y}$ is defined by $f^{\rightarrow}(A)(y)=\sup _{f(x)=y} A(x)$ for each $A \in L^{X}$, see e.g. [10], [11].

Now we are ready to define the morphisms for the category of many-valued closure spaces:

Definition 3.2 Given an L-fuzzy closure space $\left(X, c_{X}\right)$ and an $M$-fuzzy closure space $\left(Y, c_{Y}\right)$ a morphism from $\left(X, c_{X}\right)$ to $\left(Y, c_{Y}\right)$ is defined as a pair $(f, \varphi)$ where $\varphi: L \rightarrow M$ is a morphism in the category of frames and $f: X \rightarrow Y$ is a mapping such that

$$
\varphi\left(f_{L} \circ c_{X}\right) \leq c_{Y} \circ f_{L}
$$

Explicitly the last property can be written also as

$$
\varphi\left(f\left(c_{X}(A)\right)\right) \leq c_{Y}(f(A))
$$

for every $A \in L^{X}$.

The category of many-valued closure spaces, its full subcategories of many-valued closure pretopological spaces and many-valued closure topological spaces will be denoted by $\mathcal{M C l}, \mathcal{M C l P T}, \mathcal{M C l T}$ respectively.

In case when $L$ is fixed and $\varphi: L \rightarrow L$ is the identity mapping we obtain important categories of $L$-fuzzy closure , $L$-fuzzy pretopological closure and $L$-fuzzy topological closure spaces. They can be described as (non-full subcategories) of $\mathbf{M C l}$ as follows:
Definition 3.3 Let $L$ be fixed. Given two $L$ fuzzy closure spaces $\left(X, c_{X}\right)$ and $\left(Y, c_{Y}\right)$ a mapping $f: X \rightarrow Y$ is called continuous if $f\left(c_{X}(A)\right) \leq$ $c_{Y}(f(A))$ for all $A \in L^{X}$. The categories of $L$-fuzzy closure spaces, L-fuzzy closure pretopological spaces and L-fuzzy closure topological spaces and their continuous mappings are denoted by $\mathbf{C l}(L), \operatorname{ClPT}(L)$ and $\mathbf{C I T}(L)$ respectively.

\subsection{Many-valued closure spaces viewed as many-valued expansive relations}

The aim of this subsection is to characterize the category of many-valued closure spaces $\mathbf{M C l}$ as a certain subcategory $\mathcal{M R E L}(\mathbb{S}, \mathbb{F})^{1}$ of the category $\mathcal{M R E L}$ of many-valued relations. The objects of $\mathcal{M R E L}(\mathbb{S}, \mathbb{F})$ are $L$-fuzzy relations between elements of a set $X$ and $L$-fuzzy subsets of the same set $X$, that is $L$-fuzzy relations $R: X \times L^{X} \rightarrow L$. We refer to them as many-valued relations between sets and powersets of fuzzy subsets, or $L$-valued relations between sets and $L$-(fuzzy) powersets if $L$ is fixed. We define the morphisms in this category as follows.

Let many-valued relations $R: X \times L^{X} \rightarrow L$ and $S: Y \times M^{Y} \rightarrow M$ be given and let $f: X \rightarrow Y$ be a mapping and $\varphi: L \rightarrow M$ be a morphism in FRM. We define a morphism from $R: X \times L^{X} \rightarrow L$ to $S: Y \times M^{Y} \rightarrow M$ as the triple,

$$
\left(f, \varphi\left(f^{\rightarrow}\right), \varphi\right):\left(X, L^{X}, L, R\right) \rightarrow\left(Y, M^{Y}, M, S\right),
$$

in case it fulfills the condition which all morphisms in $\mathcal{M R E L}$, must satisfy, that is

$$
\varphi(R(x, A)) \leq S\left(f(x), \varphi\left(f^{\rightarrow}(A)\right)\right.
$$

for all $A \in L^{X}$, and all $x \in X$ :

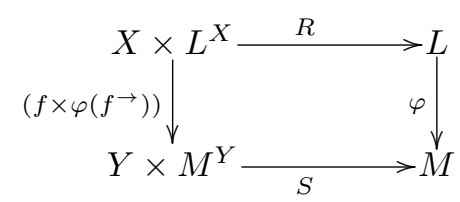

Assume now that we restrict our interest to the case of those many-valued relations

$R: X \times L^{X} \rightarrow L$, which are non-decreasing, that is

- $A_{1}, A_{2} \in L^{X}, A_{1} \leq A_{2} \Longrightarrow R\left(x, A_{1}\right) \leq$ $R\left(x, A_{2}\right)$ for all $x \in X$

and which are expansive, that is

- $R(x, A) \geq A(x)$ for all $x \in X$.

Then it is easy to see that by setting $c_{X}^{R}(A)=$ $R(x, A)$ we define an $L$-fuzzy closure operator $c_{X}^{R}$ : $L^{X} \rightarrow L^{X}$. Conversely, let $\left(X, c_{X}\right)$ be an $L$ fuzzy closure space. Then, by setting $R^{c}(x, A)=$ $c_{X}(A)(x)$ we define a non-decreasing expansive $L$ fuzzy relation $R^{c}: X \times L^{X} \rightarrow L$.

\footnotetext{
${ }^{1}$ The symbols $\mathbb{S}$ and $\mathbb{F}$ stand to recall the categories SET and FRM, respectively.
} 
Proposition 3.4 Let $R: X \times L^{X} \rightarrow L$ and $S: Y \times$ $M^{Y} \rightarrow M$ be many-valued non-decreasing expansive relations. If $\left(f, \varphi\left(f^{\rightarrow}\right), \varphi\right):\left(X, L^{X}, L, R\right) \rightarrow$ $\left(Y, M^{Y}, M, S\right)$, is a morphism in the category $\mathcal{M R E L}(\mathbb{S}, \mathbb{F})$, then $(f, \varphi):\left(X, c_{X}^{R}\right) \rightarrow\left(Y, c_{Y}^{S}\right)$ is a morphism in the category $\mathbf{M C l}$. Conversely, let $\left(X, c_{X}\right)$ be an L-fuzzy closure space and $\left(Y, c_{Y}\right)$ be an $M$-fuzzy closure space and let $\varphi: L \rightarrow M$ be a morphism of frames. If $(f, \varphi):\left(X, c_{X}\right) \rightarrow\left(Y, c_{Y}\right)$ is a morphism in the category $\mathbf{M C l}$ of many-valued closure spaces, then $(f, \varphi(f \rightarrow), \varphi)$ is a morphism in the category $\mathcal{M} \mathbf{R E L}(\mathbb{S}, \mathbb{F})$ :

Proof. The condition that

$$
\left(f, \varphi\left(f^{\rightarrow}\right), \varphi\right):\left(X, L^{X}, L, R\right) \rightarrow\left(Y, M^{Y}, M, S\right),
$$

is a morphism in the category $\mathcal{M} \mathbf{R E L}(\mathbb{S}, \mathbb{F})$ means that for every $x \in X$ and every $A \in L^{X}$ :

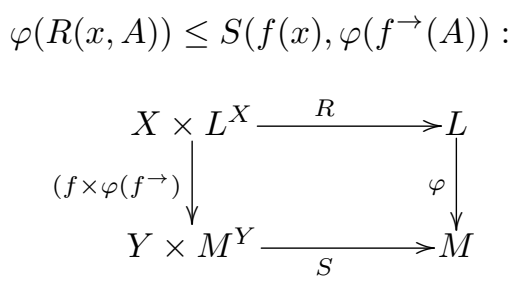

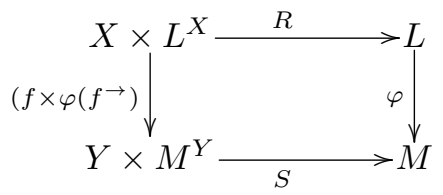

Recalling the definition of a multi-valued closure operator we get that this condition can be rewritten as

$$
\varphi\left(c_{X}(A)(x)\right) \leq c_{Y}(\varphi(f(A)))(f(x))
$$

Now since $c_{X}(A)(x)=f\left(c_{X}(A)(f(x))\right)$ we rewrite the last inequality as

$$
\varphi\left(c_{X}(f(A))\right)\left(f(x) \leq c_{Y}(\varphi(f(A))(f(x))),\right.
$$

and this just means that $(f, \varphi)$ is a morphism in the category of many-valued closure spaces. The converse can be proved in a similar way.

\section{The category of many-valued interior spaces as a category of many-valued relations}

\section{1. $L$-fuzzy interior spaces}

An alternative way to connect relations with topological type structures is via interior-type operator. Similar to the case of closure-type operators, different versions of interior type operators were used (under different names) by many researchers, see e.g. [7], [18], [8], [16], etc.

Definition 4.1 $A$ (L-fuzzy) interior operator on the L-powerset $L^{X}$ of a set $X$ is a mapping $i: L^{X} \rightarrow$ $L^{X}$ such that

(1) $i\left(1_{X}\right)=1_{X}$;

(2) $i(A) \leq A$ for every $A \in L^{X}$;

(3) $i(A) \leq i(B)$ whenever $A \leq B, A, B \in L^{X}$.

An (L-fuzzy) interior operator $i: L^{X} \rightarrow L^{X}$ is called pretopological if it satisfies condition
(4) $i(A \wedge B)=i(A) \wedge i(B)$

An (L-fuzzy) pretopological closure operator $i$ : $L^{X} \rightarrow L^{X}$ is called topological if it satisfies condition

(5) $i(i(A))=i(A)$ for every $A \in L^{X}$.

The pair $(X, i)$ is called respectively an L-fuzzy interior space, an L-fuzzy pretopological interior space, and an L-fuzzy topological interior space. In case when the frame $L$ is not specified we speak about many-valued interior operators, many-valued interior spaces, etc.

To define the category whose objects are arbitrary many-valued interior spaces and its subcategories we have to specify morphisms between such spaces. However beforehand we again introduce some notations.

Given a mapping $G: M^{Y} \rightarrow M^{X}$, where $X, Y$ are sets, and a function $\psi: M \rightarrow L$, we call preimage of $G$ under $\psi$ the function $\psi(G): M^{Y} \rightarrow L^{X}$ defined by $\psi(G)(B)(x)=\varphi(G(B)(x))$ for every $B \in M^{Y}$ and $x \in X$. Further, recall that given a mapping $f: X \rightarrow Y$, its backward $M$-powerset lift $f^{\leftarrow}$ : $M^{Y} \rightarrow M^{X}$ is defined by $f^{\leftarrow}(B)(x)=B(f(x))$ see e.g. [10], [11].

Definition 4.2 Given an L-fuzzy interior space $\left(X, i_{X}\right)$ and an $M$-fuzzy interior space $\left(Y, i_{Y}\right), a$ morphism from $\left(X, i_{X}\right)$ to $\left(Y, i_{Y}\right)$ is defined as a pair $(f, \psi)$ where $\psi: M \rightarrow L$ is a morphism of frames and $f: X \rightarrow Y$ is a mapping such that

$$
\psi\left(f_{M}^{\leftarrow} \circ i_{Y}\right) \leq i_{X} \circ \psi\left(f_{M}^{\leftarrow}\right)
$$

Equivalently the last inequality can be written as

$$
\psi\left(f^{-1}\left(i_{Y}(B)\right)\right) \leq i_{X}\left(\psi\left(f^{-1}(B)\right)\right) \forall B \in M^{Y} .
$$

The category of many-valued interior spaces, its full subcategories of many-valued pretopological interior spaces and of many-valued interior topological spaces will be denoted by $\mathcal{N}$ Int, $\mathcal{N}$ IntPT and $\mathcal{N}$ IntT respectively.

Remark 4.3 Let $L$ be fixed, and let $\left(X, i_{X}\right)$ and $\left(Y, i_{Y}\right)$ be $L$-fuzzy interior spaces. Let us consider here only morphisms of the kind $\left(f, i d_{L}\right)$ : $\left(X, i_{X}\right) \rightarrow\left(Y, i_{Y}\right)$ that is the morphisms the second component in which is the identity mapping. In this case the condition

$$
\psi\left(f_{M}^{\overleftarrow{M}} \circ i_{Y}\right) \leq i_{X} \circ \psi\left(f_{M}^{\overleftarrow{M}}\right)
$$

can be simplified as

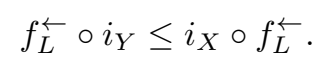

We call a mapping $f: X \rightarrow Y$ satisfying this condition continuous.

Categories of $L$-fuzzy interior, $L$-fuzzy pretopological interior and $L$-fuzzy topological interior spaces and their continuous mappings $\operatorname{Int}(L)$, $\operatorname{IntPT}(L)$ and $\operatorname{IntT}(L)$ can be found in the works of different authors, see e.g. [16] 


\subsection{Many-valued interior spaces viewed as many-valued inclusive relations}

To find connections between many-valued interior spaces and corresponding many-valued relations we start with the category $\mathcal{N R E L}$ and, first, we have to restrict the class of its objects and then to revise its morphisms.

In this subsection, as in Subsection 3.2 we consider $L$-fuzzy relations between elements of a set $X$ and $L$-fuzzy subsets of the set $X$, that is $L$-fuzzy relations $R: X \times L^{X} \rightarrow L$ (here the set $X$ and the frame $L$ are not fixed). However, as different from the approach outlined in Subsection 3.2, here we will realize our approach in the framework of the category $\mathcal{N R E L}$ instead of the category $\mathcal{M R E L}$. To define the corresponding subcategory $\mathcal{N R E L}(\mathbb{S}, \mathbb{F})$ of the category $\mathcal{N} \mathbf{R E L}$, we restrict the class of objects by taking only tuples $\left(X, L^{X}, L, R\right)$ that is $L$ fuzzy relations $R: X \times L^{X} \rightarrow L$. Further, we define the morphisms from $\left(X, L^{X}, L, R\right)$ to $\left(Y, M^{Y}, M, S\right)$ as follows.

as the triples

$$
\left(f, \psi\left(f^{\leftarrow}\right), \psi\right):\left(X, L^{X}, L, R\right) \rightarrow\left(Y, M^{Y}, M, S\right),
$$

if the condition, which all morphisms in $\mathcal{N R E L}$ must satisfy, holds, that is

$$
\psi(S(f(x), B)) \leq R\left(x, \psi\left(f^{\leftarrow}(B)\right)\right.
$$

for all $B \in M^{Y}$, and for all $x \in X$ :

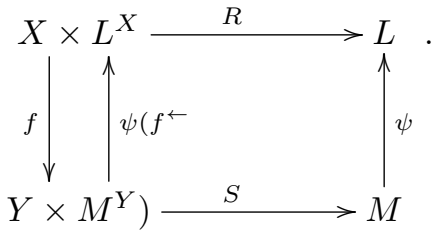

To proceed with the research of many-valued interior spaces in the framework of the category of many-valued relations in the sequel we restrict ourselves to the case of those many-valued relations $R: X \times L^{X} \rightarrow L$, which are non-decreasing, that is

- $A_{1}, A_{2} \in L^{X}, A_{1} \leq A_{2} \Longrightarrow R\left(x, A_{1}\right) \leq$ $R\left(x, A_{2}\right)$

and satisfy the condition

- $R(x, A) \leq A(x)$ for each $x \in X$.

(for convenience we call such $L$-relations inclusive.) Then one can easily notice that by setting $i_{X}^{R}(A)=$ $R(x, A)$ we define an $L$-fuzzy interior operator $i_{X}^{R}$ : $L^{X} \rightarrow L^{X}$. Conversely, if $\left(X, i_{X}\right)$ is an $L$-fuzzy interior space, then, by setting $R^{i}(x, A)=i_{X}(A)(x)$ we define a non-decreasing inclusive $L$-fuzzy relation $R^{i}: X \times L^{X} \rightarrow L$.

Proposition 4.4 Let $R: X \times L^{X} \rightarrow L$ and $S: Y \times M^{Y} \rightarrow M$ be many-valued inclusive non-decreasing relations. If $\left(f, \psi\left(f^{\leftarrow}\right), \psi\right)$ :
$\left(X, L^{X}, L, R\right) \rightarrow\left(Y, M^{Y}, M, S\right)$ is a morphism in the category $\mathcal{N} \mathbf{R E L}(\mathbb{S}, \mathbb{F})$, then $(f, \psi):\left(X, i_{X}^{R}\right) \rightarrow$ $\left(Y, i_{Y}^{S}\right)$ is a morphism in the category of manyvalued interior spaces. Let now $\left(X, i_{X}\right)$ be an $L$ fuzzy interior space and $\left(Y, i_{Y}\right)$ be an $M$-fuzzy interior space and $\psi: M \rightarrow L$ be a morphism of frames. If $(f, \psi):\left(X, i_{X}\right) \rightarrow\left(Y, i_{Y}\right)$ is a morphism in the category $\mathcal{N}$ INT, then

$$
\left(f, \psi\left(f^{\leftarrow}\right), \psi\right):\left(X, L^{X}, L, R\right) \rightarrow\left(Y, M^{Y}, M, S\right)
$$

is a morphism in the category $\mathcal{N} \mathbf{R E L}(\mathbb{S}, \mathbb{F})$.

Proof. The condition that

$$
\left(f, \psi\left(f^{\leftarrow}\right), \psi\right):\left(X, L^{X}, L, R\right) \rightarrow\left(Y, M^{Y}, M, S\right),
$$

is a morphism in $\mathcal{N} \mathbf{R E L}(\mathbb{S}, \mathbb{F})$ means that the inequality

$$
\psi(S(f(x), B)) \leq R\left(x, \psi \circ\left(f^{\leftarrow}(B)\right)\right.
$$

holds for all $B \in M^{Y}$ and for all $x \in X$. Applying definition of the many-valued interior operator induced by many-valued relations $R$ and $S$, we rewrite the above condition as

$$
\psi\left(i_{Y}(B)(f(x))\right) \leq i_{X}\left(\psi\left(f^{-1}(B)\right)\right)(x) .
$$

Now, recalling the definition of the preimage of a fuzzy set under a mapping, we rewrite the previous inequality as

$$
\psi\left(f^{-1}\left(i_{Y}(B)\right)((x))\right) \leq i_{X}\left(\psi\left(f^{-1}(B)\right)\right)(x) \forall x \in X,
$$

and hence

$$
\psi\left(f^{-1}\left(i_{Y}(B)\right) \leq i_{X}\left(\psi\left(f^{-1}(B)\right)\right)(x) \forall B \in M^{Y} .\right.
$$

However this just means that $(f, \psi):\left(X, i_{X}^{R}\right) \rightarrow$ $\left(Y, i_{Y}^{S}\right)$ is a morphism in the category of manyvalued interior spaces.

\section{Conclusions}

Basing on the concept of an $L$-fuzzy relation, wellknown to people working in "Fuzzy Mathematics", we introduced two categories of many-valued relations, that is $L$-fuzzy relations where the range $L$ is not fixed. Our special interest here was in the description of different approaches to the subject of Fuzzy Topology in the framework of the categories of many-valued relations. This description was presented in two parallel ways; the first one is based on closure-type operators, while the second one as the basic takes interior-type operators.

As the main perspectives for the future work we see the following.

First, we expect that the study of the categories $\mathcal{M R E L}$ and $\mathcal{N}$ REL themselves will be done. In particular, operations of product, co-product and quotients in these categories should be constructed and their relation to the corresponding operations of fuzzy topological spaces should be studied. 
Second, it would be interesting and useful to characterize special topological properties, like compactness, separation axioms, etc. of fuzzy topological spaces in terms of many-valued relations generating these spaces.

Finally, we assume that Fuzzy Topology is not the only area where many-valued relations can be useful. In particular, at present we are working on the description of categories of fuzzy soft spaces and fuzzy rough spaces in the framework of many-valued relations.

Acknowledgment The second named author kindly announces the support of the ESF project 2013/0024/1DP/1.1.1.2.0/13/APIA/VIAA/045

The authors are also grateful to the three referees for reading the paper carefully and making constructive critical remarks.

\section{References}

[1] U. Bodenhofer, Ordering of fuzzy sets based on fuzzy orderings. I: The basic approach. Mathware Soft Comput. 15 (2008) 201-218.

[2] U. Bodenhofer, Ordering of fuzzy sets based on fuzzy orderings. II: Generalizations. Mathware Soft Comput. 15 (2008) 219-249.

[3] P.N. Cohn, Universal algebra, Harper and Row Publ., New York, 1965.

[4] D. Dubois, H. Prade, Rough fuzzy sets and fuzzy rough sets, Internat. J. General Systems 17 (2-3) (1990) 191-209.

[5] B.A.Davey, H.A. Priestly, Introduction to Lattices and Order, Second edition. Cambridge University Press, 2002.

[6] G. Gierz, K.H. Hoffman, K. Keimel, J.D. Lawson, M.W. Mislove, D.S. Scott, Continuous Lattices and Domains, Cambridge University Press, Cambridge, 2003.

[7] Liu Ying-Ming, Luo Mao-Kang, Fuzzy topology. Advances in Fuzzy Systems - Applications and Theory 9. World Scientific, Singapore, 1997.

[8] U. Höhle, A.Šostak, Axiomatic foundations of fixed-based fuzzy topology, in: U.Höhle, S.E. Rodabaugh (Eds.) Mathematics of Fuzzy Sets: Logic, Topology and Measure Theory, Kluwer Acad. Publ. (1999) 123-273.

[9] M. Erne, Lattice representations for categories of closure spaces, in: Categorical Topology. Sigma Series in Pure Mathematics 5. Helderman Verlag Berlin, 1983., 197-223.

[10] S.E. Rodabaugh, Powerset operator based foundations for point-set lattice-theoretic (poslat) fuzzy set theories and topologies, Quaest. Math. 20 (1997) 463-530.

[11] S.E. Rodabaugh, Powerset operator foundations for poslat fuzzy set theories and topologies, in: U.Höhle, S.E. Rodabaugh (Eds.) Mathematics of Fuzzy Sets: Logic, Topol- ogy and Measure Theory, Kluwer Acad. Publ. (1999) 91-116.

[12] S.E. Rodabaugh, Categorical foundations of variable-based fuzzy topology, in: U.Höhle, S.E. Rodabaugh (Eds.) Mathematics of Fuzzy Sets: Logic, Topology and Measure Theory, Kluwer Acad. Publ. (1999) 91-116.

[13] A. Šostak, Basic structures of fuzzy topology, J. Math. Sci 78:6 (1996), 662-701.

[14] A. Šstak, Towards the theory of $M$ approximate systems: Fundamentals and examples, Fuzzy Sets and Syst. 161 (2010) 2440-2461.

[15] A. Šostak, Towards the theory of approximate systems: variable range categories. Proceedings of ICTA2011, Islamabad, Pakistan. Cambridge University Publ. (2012) 265-284.

[16] A. Šostak, On some fuzzy categories related to category L-Top of L-topological spaces, Chapter 12 In: Topological and algebraic structures in fuzzy sets. S.E. Rodabaugh and E.P. Klement eds, Kluwer Academic Publ., Dodrecht, Boston, London, 2003, pp. 337-371.

[17] L. Valverde, On the structure of $F$ indistinguishibility operators, Fuzzy Sets and Syst. 17 (1985) 313-328.

[18] R.H. Warren, Neighborhoods, bases and continuity in fuzzy topological spaces, Rocky Mount. J. Math. 8 (1978), 459-470.

[19] L. Zadeh Similarity relations and fuzzy orderings, Inf. Sci. 3 (1971) 177-200. 